\title{
La toma de decisiones compartidas desde la perspectiva del paciente oncológico: roles de participación y valoración del proceso
}

\section{Shared decision making from the perspective of the cancer patient: participatory roles and evaluation of the process}

doi.org/10.23938/ASSN.0003

\author{
N. Padilla-Garrido ${ }^{1}$, F. Aguado-Correa ${ }^{2}$, M. Ortega-Moreno ${ }^{1}$, J. Bayo-Calero ${ }^{3}$, E. Bayo-Lozano ${ }^{4}$
}

\section{RESUMEN}

Fundamento. En España no existe un claro conocimiento sobre el grado en que la Toma de Decisiones Compartidas (TDC) es llevada a la práctica habitual en oncología. Nuestro trabajo analiza el rol preferido y el rol percibido de los pacientes oncológicos y mide el proceso de TDC desde su perspectiva.

Material y métodos. Estudio descriptivo transversal mediante cuestionario autoadministrado a pacientes con distintos tipos de cáncer. Para evaluar el rol preferido y percibido por el paciente utilizamos The Control Preference Scales (CPS) y para medir la TDC se utilizó The nineitem Shared Decision Making Questionnaire (SDM-Q-9).

Resultados. De los 132 pacientes encuestados, solo 118 proporcionaron datos analizables. No se encontraron evidencias de que el sexo, edad, nivel educativo o tipo de tumor afectaran al rol preferido o al percibido. Solo el 59,3\% estaba de acuerdo con el rol ejercido. Todos los que preferían un rol pasivo lo alcanzaban $(21,2 \%)$, mientras que de los que deseaban uno compartido (78,8\%), solo el $48,39 \%$ lo lograba y el $51,61 \%$ restante desempeñaba uno pasivo. Ninguno prefirió ni ejerció un rol activo. El conjunto de pacientes valoró el proceso de TDC con una puntuación de $41,07 \pm 5,94$, en una escala de 0 a 100, alcanzando los pacientes urológicos una puntuación máxima de $61,39 \pm 13,24$.

Conclusiones. Nuestro estudio no encuentra evidencias de que, desde el punto de vista del paciente oncológico, el modelo de TDC esté implementado en la práctica.

Palabras clave: Cáncer. Toma de decisiones. Satisfacción del paciente. Participación del paciente. Rol.

\begin{abstract}
Background. In Spain there is no clear knowledge about the degree to which Shared Decision Making (SDM) is carried out in the normal practice of oncology. Our article analyses the preferred role and the perceived role of oncological patients and measures the SDM process from their perspective.
\end{abstract}

Material and methods. Descriptive transversal study using a self-conducted questionnaire with patients with different types of cancer. To evaluate the role preferred and perceived by the patient we used The Control Preference Scales (CPS) and to measure SDM we used The nine-item Shared Decision Making Questionnaire (SDM-Q-9).

Results. Out of the 132 patients surveyed, only 118 provided analysable data. No evidence was found that sex, age, educational level or type of tumour affected the preferred role or the perceived role. Only $59.3 \%$ was in agreement with the role exercised. All of those who preferred a passive role achieved this (21.2\%), while out of those who wanted a shared role $(78.8 \%)$, this was achieved by only $48.39 \%$ while the remaining $51.61 \%$ played a passive role. None preferred or played an active role. The set of patients evaluated the SDM process with a score of $41.07 \pm 5.94$, on a scale of 0 to 100 , with the highest score of $61.39 \pm 13.24$ reached by urological patients.

Conclusions. Our study found no evidence that, from the point of view of the oncological patient, the SDM model is being implemented in practice.

Keywords: Neoplasms. Decision making. Patient satisfaction. Patient participation. Role.
1. Departamento de Métodos Cuantitativos para la Economía y la Empresa. Estadística e Investigación Operativa. Universidad de Huelva.

2. Departamento de Economía Financiera, Contabilidad y Dirección de Operaciones. Universidad de Huelva.

3. Servicio de Oncología Médica. Hospital Juan Ramón Jiménez. Huelva.

4. Servicio de Oncología Radioterápica. Hospital Juan Ramón Jiménez. Huelva.

Recepción: 21/04/2016

Aceptación provisional: 26/07/2016

Aceptación definitiva: 27/12/2016

\section{Correspondencia:}

N. Padilla-Garrido

Departamento de Métodos Cuantitativos para la

Economía y la Empresa, Estadística e Investigación Operativa

Universidad de Huelva

Plaza de la Merced, 11

21071 Huelva

E-mail: padilla@uhu.es 


\section{INTRODUCCIÓN}

En numerosas ocasiones, pacientes y médicos se enfrentan a situaciones en las que deben elegir entre más de una opción diagnóstica o terapéutica razonable desde el punto de vista médico. Cada opción tiene sus ventajas e inconvenientes siendo imposible encontrar la mejor. Precisamente en estas circunstancias en las que no existe una decisión que supere a las demás es donde se hace imprescindible la participación del paciente en la toma de decisiones ${ }^{1}$ a través de la incorporación de sus valores y preferencias.

En este contexto, y en conjunción con un creciente interés por la atención centrada en el paciente, se acuña por primera vez en 1982 el término Toma de Decisiones Compartidas ${ }^{2}$ (TDC). La TDC se define como un proceso de decisión conjunto entre médico y paciente. Se inicia con la información ofrecida por el profesional al paciente sobre las diferentes alternativas disponibles, las posibles consecuencias asociadas a cada una de ellas así como sus ventajas e inconvenientes. Posteriormente, el paciente aporta sus valores y preferencias llegando, finalmente, a un acuerdo sobre la opción a elegir.

Diversos estudios han demostrado los beneficios que la TDC puede aportar a los pacientes entre los cuales destacan la disminución del miedo y la depresión, la mejora de la calidad de vida o el aumento de la satisfacción con el tratamiento ${ }^{3-5}$.

$\mathrm{Su}$ aplicación es especialmente útil en las consultas oncológicas, como, por ejemplo, en el caso del cáncer de mama y la decisión entre tumorectomía o mastectomía, o en el de próstata y la elección entre vigilancia activa, cirugía o radioterapia $^{6,7}$.

Aunque en los últimos años la TDC está adquiriendo notoriedad, es necesario saber hasta qué punto este modelo se está implementando en la práctica. En este sentido, y desde la perspectiva del paciente, es posible medir varios aspectos como: 1) el rol que el enfermo prefiere adoptar en los antecedentes de la decisión, 2) el proceso de toma de decisiones en sí y, 3) el rol que el paciente percibe que ha desarrollado durante el encuentro clínico.

Con respecto al primero, el paradigma de la TDC está directamente relacionado con la preferencia del paciente por un rol compartido o colaborativo frente a otro tipo de roles como el activo, en caso de que el paciente prefiera tomar la decisión, o el pasivo, si prefiere que sea el médico el que decida. Sin embargo, a lo largo de la literatura no existe una clara evidencia de que los pacientes oncológicos prefieran ejercer un rol compartido. Así mientras que algunas revisiones han mostrado su preferencia por adoptar un rol compartido o activo $^{8}$, otras, en cambio, señalan la preferencia por un rol pasivo ${ }^{9}$.

En referencia al rol percibido, también hay trabajos que evidencian que los pacientes oncológicos, a menudo, no alcanzan el nivel deseado de participación en el proceso de toma de decisiones. Así, una revisión sistemática de estudios con enfermos de diferentes tipos de cáncer ha constatado que la mayoría de ellos prefería una mayor implicación de la que realmente percibían ${ }^{10}$. Esta discrepancia entre rol preferido y rol percibido puede tener importantes consecuencias como provocar una menor satisfacción con la decisión ${ }^{11}$, unos mayores niveles de depresión ${ }^{11} \mathrm{o}$ una inferior calidad de vida física y emocional ${ }^{12}$.

Por último y con respecto al grado en que el paciente piensa que el proceso de TDC se ha implementado en la práctica, existen diferentes instrumentos de medi$\mathrm{da}^{13}$ de cuya calidad dependerá, finalmente, la eficacia de la evaluación.

Por lo que respecta a España, la investigación sobre TDC se encuentra en una etapa temprana ${ }^{14}$. Los estudios que podríamos relacionar con los roles del paciente no los analizan como tales, sino que se centran en la participación del enfermo en la toma de decisiones. En esta línea, las investigaciones se han desarrollado, principalmente, en el campo de la atención primaria y la oncología. En el primer ámbito, los estudios revelan que, aunque la participación del 
paciente sea escasa ${ }^{15,16}$, existe un deseo del mismo por intervenir de alguna forma ${ }^{16-18}$. Por su parte, en oncología se obtienen conclusiones en la misma línea ${ }^{19}$, evidenciando que, en la mayoría de los casos, el paciente con cáncer delega la responsabilidad de la decisión al médico, aunque existe una tendencia hacia un mayor deseo de participación. Con respecto a la medición del proceso de TDC, no existen estudios en el ámbito de la oncología. Solo hay un trabajo que compara los resultados obtenidos por pacientes psiquiátricos y de atención pri$\operatorname{maria}^{20}$.

Por tanto, y dado que no existe un claro conocimiento sobre el grado en que la TDC es llevada a la práctica habitual en el campo de la oncología en España, nuestro estudio tiene como objetivo principal analizar el rol preferido y el rol percibido por el paciente con cáncer en la toma de decisiones sobre su tratamiento así como medir el proceso de TDC desde su perspectiva. Como objetivo secundario, analizaremos la concordancia entre roles así como si éstos y la medición resultante de la TDC están influenciados por el tipo de tumor que padece el enfermo.

\section{MATERIAL Y MÉTODOS}

Se realizó un estudio descriptivo transversal mediante cuestionario autoadministrado, en el periodo comprendido entre noviembre de 2014 y enero de 2015.

Los participantes en el estudio fueron 132 pacientes oncológicos, mayores de edad, atendidos en el hospital público Juan Ramón Jiménez de Huelva que se habían enfrentado con su médico a una toma de decisiones sobre su tratamiento con distintas alternativas apropiadas y que ofrecían la misma posibilidad de superar la enfermedad a largo plazo. De todos ellos, solo 118 proporcionaron datos analizables. El estudio no tuvo financiación y antes de comenzarlo se obtuvo la aprobación del Comité de Ética del hospital.

La captación de los participantes se llevó a cabo a través de profesionales sani- tarios de diferentes consultas oncológicas mediante muestreo casual y periodos de tiempo seleccionados aleatoriamente. De este modo, cada uno de ellos eligió, directa e intencionadamente, a los pacientes que convivían con la patología desde hacía algún tiempo y que se habían enfrentado a una decisión sobre el tratamiento eligiendo entre más de una opción, garantizando, de este modo, que se cumplía el requisito necesario para el estudio.

Una vez realizada la invitación por parte del médico, cada participante recibió una explicación detallada por escrito del estudio y, tras firmar su consentimiento informado, completó un cuestionario anónimo sin la presencia de profesionales sanitarios y sin recibir compensación económica por ello.

Para ello, se instruyó a los participantes con el propósito de que usaran como referencia la consulta en la que habían realizado la TDC. Dado que no todos los pacientes estaban familiarizados con el término TDC, en cada encuesta se proporcionó una de las definiciones más populares de la misma elaborada por Charles y col ${ }^{21}$. El cuestionario incluía cuatro partes: 1) información sociodemográfica, 2) información clínica, 3) rol preferido y rol percibido en la toma de decisiones y 4) medición de la TDC.

El apartado de información sociodemográfica englobaba sexo, edad y nivel educativo (estudios universitarios, secundarios, primarios y sin estudios).

La Información clínica incluía el tipo de tumor que padecía el paciente. Éste se clasificó en tres posibles grupos (cáncer de mama, urológico y otros), los cuales se establecieron en función del riesgo relativo suavizado de muerte de los municipios de la provincia de Huelva ${ }^{22}$.

En el apartado de roles, para evaluar el rol preferido por el paciente en la toma de decisiones sobre su tratamiento oncológico se utilizó la adaptación al español realizada por Peralta ${ }^{16}$ de The Control Preference Scales $^{23}$ (CPS), instrumento validado y utilizado por pacientes con cáncer en estudios previos $^{24}$. En este sentido, a los pacientes se les preguntó cuál, de los siguientes cin- 
co posibles estados, reflejaba mejor el rol que preferían en los antecedentes de la decisión:

a) Prefiero tomar yo mismo la decisión final sobre el tratamiento que debo seguir.

b) Prefiero tomar yo mismo la decisión final sobre el tratamiento que debo seguir después de considerar la opinión de mi médico.

c) Prefiero que mi médico y yo compartamos la responsabilidad de decidir qué tratamiento es mejor para mí.

d) Prefiero que mi médico tome la decisión final sobre el tratamiento que debo seguir, pero después de considerar mi opinión.

e) Prefiero dejar la decisión final sobre el tratamiento que debo seguir exclusivamente en manos de mi médico.

Las dos primeras respuestas indicaban una preferencia por un rol activo, la tercera por uno compartido y las dos últimas por uno pasivo. La misma escala se utilizó también para registrar el rol percibido tras la toma de decisiones. De esta forma, se podían hacer comparaciones entre los roles preferidos y percibidos, indicando la discrepancia entre ellos.

Por último, para medir el proceso de TDC se utilizó la versión española de The nine-item Shared Decision Making Questionnaire (SDM-Q-9) ${ }^{25}$, adaptada y validada por De las Cuevas y $\mathrm{col}^{26}$. Se trata de un cuestionario breve y simple que permite evaluar la TDC desde la perspectiva del paciente, teniendo en cuenta los pasos que intervienen en la misma, y que ha demostrado buena aceptabilidad, fiabilidad y valide $z^{25,26}$.

A los pacientes se les preguntó con respecto a nueve afirmaciones relacionadas con la experiencia que tuvo en la consulta con su médico y que describen los pasos del proceso de TDC. Cada uno de estos ítems puede puntuarse en una escala de Likert de 6 puntos, con un rango que va desde "totalmente en desacuerdo" (0) hasta "totalmente de acuerdo" (5). La suma de las respuestas a los nueve ítems genera una puntuación total del paciente comprendida entre 0 y 45. Para facilitar su interpretación, dicha puntuación se multiplica por $20 / 9$ y se obtiene una puntuación transformada entre 0 y 100 , donde 0 indica el peor nivel posible en la TDC y 100 el mejor ${ }^{26}$.

\section{Análisis estadístico de los datos}

Se hizo un análisis descriptivo a partir de las frecuencias absolutas y porcentajes de las variables sexo, edad, nivel educativo, tipo de tumor, rol real y rol preferido. Para contrastar la existencia o no de relación entre sexo, edad, nivel educativo y tipo de tumor con respecto al rol preferido y rol percibido fueron utilizados el test $\chi^{2}$ de asociación o, en caso necesario, el test exacto de Fisher.

Además, se compararon ambos tipos de roles en cada paciente para saber si existía concordancia o no entre ellos. La prueba de McNemar se utilizó para determinar si había diferencias significativas entre ambos.

Con respecto al SDM-Q-9 se realizó un análisis inicial para conocer la puntuación transformada del conjunto de pacientes así como por tipo de tumor, empleando el test de Kruskall-Wallis para comprobar si existían diferencias entre los distintos grupos de cáncer, y, en caso afirmativo, la prueba de U de Mann-Whitney, con corrección de Bonferroni, para analizar, por pares, qué tipos de cáncer diferían entre sí.

También, y con el fin de analizar la orientación de las respuestas del SDM-Q-9 ítem por ítem, las posibles respuestas se agruparon en dos categorías: "Desacuerdo" y "Acuerdo". La primera de estas categorías englobaba a: "Totalmente en desacuerdo", "Muy en desacuerdo" y "Algo en desacuerdo", mientras que la segunda incluía a: "Algo de acuerdo", "Muy de acuerdo" y "Totalmente de acuerdo". Se contabilizó el número total y el porcentaje de pacientes que estaban de acuerdo o en desacuerdo con las afirmaciones de cada uno de los 9 ítems. También se hizo el mismo análisis por tipo de tumor.

El software empleado fue SPSS Versión 20.0 . 


\section{RESULTADOS}

Los resultados totales de las características sociodemográficas y clínicas se recogieron en la tabla 1. Para realizar el análisis, la variable edad fue dividida en función de la mediana. Junto a los totales, en dicha tabla se ofrecen los resultados correspondientes al rol preferido y al rol percibido. Aunque cuando a los pacientes se le preguntó por los roles, estos podían optar por cinco posibilidades, ninguno seleccionó las respuestas $\mathrm{A}$ o $\mathrm{B}$ correspondientes a un rol activo.

La edad media de los encuestados fue de 57,97 años, siendo el 53,4\% mujeres. La mayoría contaba con estudios primarios (47,5\%) o carecía de estudios (14,4\%). El $35,6 \%$ de los casos presentaba cáncer de mama, el $20,3 \%$ urológico y el $44,1 \%$ otros tipos de cáncer.

Al contrastar las variables sociodemográficas y clínicas con respecto a ambos roles (Tabla 1), no se encontraron diferencias significativas por sexo, grupo de edad, nivel educativo y grupo de tumor.

$\mathrm{Al}$ analizar el rol preferido, el 78,8\% manifestó que deseaba uno compartido mientras que el $21,2 \%$ uno pasivo. Por lo que respecta al rol percibido, el $38,1 \%$ de los pacientes respondió que éste fue compartido y el $61,9 \%$ que fue pasivo.

De todos los pacientes, solo en un $59,3 \%$ de los casos coincidía rol preferido y percibido, destacando que todos aquellos que preferían un rol pasivo (25 pacientes), percibieron que lo habían conseguido (Tabla 2).

Tabla 1. Características y roles de los pacientes.

\begin{tabular}{|c|c|c|c|c|c|c|c|c|c|c|c|c|c|c|}
\hline & \multicolumn{2}{|c|}{ TOTALES } & \multicolumn{4}{|c|}{ ROL PREFERIDO } & \multicolumn{8}{|c|}{ ROL PERCIBIDO } \\
\hline & \multirow[b]{2}{*}{$\mathrm{N}$} & \multirow[b]{2}{*}{$\%$} & \multicolumn{2}{|c|}{ Compartido } & \multicolumn{2}{|c|}{ Pasivo } & \multirow[b]{2}{*}{$\chi^{2}$} & \multirow[b]{2}{*}{$\mathrm{P}$} & \multicolumn{2}{|c|}{ Compartido } & \multicolumn{2}{|c|}{ Pasivo } & \multirow[b]{2}{*}{$\chi^{2}$} & \multirow[b]{2}{*}{$\mathrm{P}$} \\
\hline & & & $\mathrm{N}$ & $\%$ & $\mathrm{~N}$ & $\%$ & & & $\mathrm{~N}$ & $\%$ & $\mathrm{~N}$ & $\%$ & & \\
\hline & 118 & $100 \%$ & 93 & $78,8 \%$ & 25 & $21,2 \%$ & & & 45 & $38,1 \%$ & 73 & $61,9 \%$ & & \\
\hline \multicolumn{15}{|l|}{ Sexo } \\
\hline Hombre & 55 & $46,6 \%$ & 47 & $85,5 \%$ & 8 & $14,5 \%$ & \multirow{2}{*}{2,721} & \multirow{2}{*}{0,099} & 25 & $45,5 \%$ & 30 & $54,5 \%$ & \multirow{2}{*}{2,339} & \multirow{2}{*}{0,126} \\
\hline Mujer & 63 & $53,4 \%$ & 46 & $73,0 \%$ & 17 & $27,0 \%$ & & & 20 & $31,7 \%$ & 43 & $68,3 \%$ & & \\
\hline \multicolumn{15}{|l|}{ Edad } \\
\hline Mayor o igual a 60 & 59 & $50,0 \%$ & 47 & $79,7 \%$ & 12 & $20,3 \%$ & \multirow{2}{*}{0,051} & \multirow{2}{*}{0,822} & 23 & $39,0 \%$ & 36 & $61,0 \%$ & \multirow{2}{*}{0,036} & \multirow{2}{*}{0,850} \\
\hline Menor de 60 & 59 & $50,0 \%$ & 46 & $78,0 \%$ & 13 & $22,0 \%$ & & & 22 & $37,3 \%$ & 37 & $62,7 \%$ & & \\
\hline \multicolumn{15}{|l|}{ Nivel educativo } \\
\hline Estudios universitarios & 16 & $13,6 \%$ & 14 & $87,5 \%$ & 2 & $12,5 \%$ & \multirow{4}{*}{$1,849^{*}$} & \multirow{4}{*}{0,601} & 7 & $43,8 \%$ & 9 & $56,3 \%$ & \multirow{4}{*}{2,774} & \multirow{4}{*}{0,425} \\
\hline Estudios secundarios & 29 & $24,6 \%$ & 24 & $82,8 \%$ & 5 & $17,2 \%$ & & & 13 & $44,8 \%$ & 16 & $55,2 \%$ & & \\
\hline Estudios primarios & 56 & $47,5 \%$ & 41 & $73,2 \%$ & 15 & $26,8 \%$ & & & 17 & $30,4 \%$ & 39 & $69,6 \%$ & & \\
\hline Sin estudios & 17 & $14,4 \%$ & 14 & $82,4 \%$ & 3 & $17,6 \%$ & & & 8 & $47,1 \%$ & 9 & $52,9 \%$ & & \\
\hline \multicolumn{15}{|l|}{ Grupo tumor } \\
\hline Mama & 42 & $35,6 \%$ & 35 & $83,3 \%$ & 7 & $16,7 \%$ & \multirow{3}{*}{3,425} & \multirow{3}{*}{0,18} & 15 & $35,7 \%$ & 27 & $64,3 \%$ & \multirow{3}{*}{5,450} & \\
\hline Otros & 52 & $44,1 \%$ & 37 & $71,2 \%$ & 15 & $28,8 \%$ & & & 16 & $30,8 \%$ & 36 & $69,2 \%$ & & 0,066 \\
\hline Urológico & 24 & $20,3 \%$ & 21 & $87,5 \%$ & 3 & $12,5 \%$ & & & 14 & $58,3 \%$ & 10 & $41,7 \%$ & & \\
\hline
\end{tabular}

* Estadístico exacto de Fisher 
Tabla 2. Comparación del rol preferido y percibido por los pacientes.

\begin{tabular}{lcccc}
\hline \multicolumn{5}{c}{ Rol percibido $(\boldsymbol{N})$} \\
\hline Rol preferido & Activo & Compartido & Pasivo & Total, $\boldsymbol{N}(\%)$ \\
\hline Activo & $0^{\mathrm{a}}$ & 0 & 0 & $0(0)$ \\
\hline Compartido & 0 & $45^{\mathrm{a}}$ & 48 & $93(78,8)$ \\
\hline Pasivo & 0 & 0 & $25^{\mathrm{a}}$ & $25(21,2)$ \\
\hline Total general, $N(\%)$ & $0(0)$ & $45(38,1)$ & $73(61,9)$ & $118(100)$ \\
\hline
\end{tabular}

$\%$ de acuerdo $=(0+45+25) / 118=59,3 \%$

a Completo acuerdo

La prueba de McNemar $\left(\chi^{2}=46,021\right.$, $\mathrm{P}<, 001)$ permitió afirmar que existían diferencias significativas entre el rol preferido y el percibido.

Por lo que respecta al SDM-Q-9, la puntuación transformada del conjunto de pacientes fue de $41,07 \pm 5,94$. Según el tipo de tumor, la puntuación de los pacientes con cáncer de mama fue de $34,81 \pm 10,27$, la del grupo otros de $36,75 \pm 8,22$ y la de los urológicos fue $61,39 \pm 13,24$.

La prueba de Kruskal-Wallis $\left(\chi^{2}=10,349\right.$, $\mathrm{p}=, 006)$ permitió afirmar que existían diferencias significativas en las puntuaciones totales de la variable que recogía el grado de valoración de la TDC según el tipo de cáncer.

La prueba de U de Mann-Whitney para muestras independientes, con corrección de Bonferroni, evidenció que las percepciones de los pacientes urológicos diferían significativamente con las de los pacientes de mama y otros tipos de cáncer. Sin embargo, no había diferencias entre pacientes de mama y otros tipos de cáncer.

Los resultados obtenidos en cada pregunta del cuestionario SDM-Q-9 se muestran en la tabla 3 , tanto globalmente como por tipo de tumor. En todos los casos se analizó el número y el porcentaje de pacientes que estaban de acuerdo y en desacuerdo con cada afirmación. En la valoración de todos los pacientes predominaba el desacuerdo, salvo en la afirmación 5 . Este patrón se repitió para los tumores de mama y otros. Sin embargo, en la valoración de los pacientes urológicos prevaleció el acuerdo, excepto en las afirmaciones 7 y 9 .

\section{DISCUSIÓN}

En nuestro análisis no se han encontrado evidencias de que el sexo, la edad, el nivel educativo o el tipo de tumor afectaran al rol preferido o al percibido por el paciente. Un elevado porcentaje de pacientes prefería un rol compartido, pero menos de la mitad percibió que lo había podido desempeñar en la consulta. Ninguno prefirió ni ejerció un rol activo. El conjunto de pacientes valoró el proceso de TDC con una puntuación por debajo del valor medio de la escala.

La falta de relación reflejada en nuestro estudio entre los factores sociodemográficos y el rol preferido de los pacientes oncológicos y también ha sido recogida en trabajos previos ${ }^{27,28}$. Sin embargo, hay estudios que demuestran que el nivel preferido de participación varía con dichos factores e, incluso, con el tipo de cáncer ${ }^{29,30}$.

Otro aspecto que también se ha destacado en la literatura, y que nosotros hemos evidenciado, es la preferencia del enfermo de cáncer por un rol compartido ${ }^{8}$, así como la diferencia existente entre el rol preferido y el percibido ${ }^{10}$.

En los estudios sobre roles, la mayoría de la literatura se centra, exclusivamente, en pacientes con una tipología de cáncer, especialmente el de mama. Por el contrario, nuestro estudio aborda pacientes con distintos tipos de cáncer, al igual que el trabajo de Singh y col ${ }^{29}$, o tres de los 22 estudios incluidos en la revisión de Tariman $\mathrm{y} \mathrm{col}^{10}$, o el de Colley y otros ${ }^{30}$. Al respecto, nuestro estudio presenta un rol preferido pasivo del $21 \%$ similar al $25 \%$ recogido por 
Tabla 3. Resultados obtenidos en los ítems del SDM-Q-9.

\begin{tabular}{|c|c|c|c|c|c|c|c|c|c|c|c|c|c|c|c|c|}
\hline \multirow{3}{*}{ SDM-Q-9 Ítems } & \multicolumn{4}{|c|}{ TOTAL } & \multicolumn{4}{|c|}{ MAMA } & \multicolumn{4}{|c|}{ OTROS } & \multicolumn{4}{|c|}{ UROLÓGICO } \\
\hline & \multicolumn{2}{|c|}{ Acuerdo } & \multicolumn{2}{|c|}{ Desacuerdo } & \multicolumn{2}{|c|}{ Acuerdo } & \multicolumn{2}{|c|}{ Desacuerdo } & \multicolumn{2}{|c|}{ Acuerdo } & \multicolumn{2}{|c|}{ Desacuerdo } & \multicolumn{2}{|c|}{ Acuerdo } & \multicolumn{2}{|c|}{ Desacuerdo } \\
\hline & $\mathrm{N}$ & $\%$ & $\mathrm{~N}$ & $\%$ & $\mathrm{~N}$ & $\%$ & $\mathrm{~N}$ & $\%$ & $\mathrm{~N}$ & $\%$ & $\mathrm{~N}$ & $\%$ & $\mathrm{~N}$ & $\%$ & $\mathrm{~N}$ & $\%$ \\
\hline $\begin{array}{l}\text { 1. Mi médico me dijo expre- } \\
\text { samente que debía tomarse } \\
\text { una decisión }\end{array}$ & 52 & $44,1 \%$ & 66 & $55,9 \%$ & 15 & $12,7 \%$ & 27 & $22,9 \%$ & 19 & $16,1 \%$ & 33 & $28,0 \%$ & 18 & $15,3 \%$ & 6 & $5,1 \%$ \\
\hline $\begin{array}{l}\text { 2. Mi médico quería saber } \\
\text { exactamente cómo me gus- } \\
\text { taría participar en la toma } \\
\text { de decisiones }\end{array}$ & 44 & $37,3 \%$ & 74 & $62,7 \%$ & 13 & $11,0 \%$ & 29 & $24,6 \%$ & 13 & $11,0 \%$ & 39 & $33,1 \%$ & 18 & $15,3 \%$ & 6 & $5,1 \%$ \\
\hline $\begin{array}{l}\text { 3. Mi médico me informó de } \\
\text { que existen distintas opcio- } \\
\text { nes de tratamiento para mi } \\
\text { problema de salud }\end{array}$ & 45 & $38,1 \%$ & 73 & $61,9 \%$ & 13 & $11,0 \%$ & 29 & $24,6 \%$ & 16 & $13,6 \%$ & 36 & $30,5 \%$ & 16 & $13,6 \%$ & 8 & $6,8 \%$ \\
\hline $\begin{array}{l}\text { 4. Mi médico me explicó } \\
\text { con exactitud las ventajas y } \\
\text { desventajas de las distintas } \\
\text { opciones de tratamiento }\end{array}$ & 52 & $44,1 \%$ & 66 & $55,9 \%$ & 14 & $11,9 \%$ & 28 & $23,7 \%$ & 20 & $16,9 \%$ & 32 & $27,1 \%$ & 18 & $15,3 \%$ & 6 & $5,1 \%$ \\
\hline $\begin{array}{l}\text { 5. Mi médico me ayudó a en- } \\
\text { tender toda la información }\end{array}$ & 104 & $88,1 \%$ & 14 & $11,9 \%$ & 35 & $29,7 \%$ & 7 & $5,9 \%$ & 45 & $38,1 \%$ & 7 & $5,9 \%$ & 24 & $20,3 \%$ & 0 & $0,0 \%$ \\
\hline $\begin{array}{l}\text { 6. Mi médico me preguntó } \\
\text { qué opción de tratamiento } \\
\text { prefiero }\end{array}$ & 35 & $29,7 \%$ & 83 & $70,3 \%$ & 9 & $7,6 \%$ & 33 & $28,0 \%$ & 11 & $9,3 \%$ & 41 & $34,7 \%$ & 15 & $12,7 \%$ & 9 & $7,6 \%$ \\
\hline $\begin{array}{l}\text { 7. Mi médico y yo valoramos } \\
\text { con detenimiento las distin- } \\
\text { tas opciones de tratamiento }\end{array}$ & 36 & $30,5 \%$ & 82 & $69,5 \%$ & 14 & $11,9 \%$ & 28 & $23,7 \%$ & 18 & $15,3 \%$ & 34 & $28,8 \%$ & 4 & $3,4 \%$ & 20 & $16,9 \%$ \\
\hline $\begin{array}{l}\text { 8. Mi médico y yo elegimos } \\
\text { juntos una opción de trata- } \\
\text { miento }\end{array}$ & 38 & $32,2 \%$ & 80 & $67,8 \%$ & 8 & $6,8 \%$ & 34 & $28,8 \%$ & 17 & $14,4 \%$ & 35 & $29,7 \%$ & 13 & $11,0 \%$ & 11 & $9,3 \%$ \\
\hline $\begin{array}{l}\text { 9. Mi médico y yo llegamos } \\
\text { a un acuerdo sobre el modo } \\
\text { de proceder }\end{array}$ & 40 & $33,9 \%$ & 78 & $66,1 \%$ & 13 & $11,0 \%$ & 29 & $24,6 \%$ & 19 & $16,1 \%$ & 33 & $28,0 \%$ & 8 & $6,8 \%$ & 16 & $13,6 \%$ \\
\hline
\end{tabular}

Singh y $\mathrm{col}^{29}$. Lo mismo ocurre en el rol percibido compartido, con un $38,1 \%$ frente a un $34 \%$. También es destacable el elevado parecido en el porcentaje de pacientes que alcanzó el nivel de participación deseado (59,3\% vs $61 \%)$.

Sin embargo, hay una clara diferencia en el rol percibido pasivo (61,9\% vs $36 \%$ ) y en el rol preferido compartido $(78,8 \%$ vs $49 \%$ ), posiblemente explicada por la llamativa inexistencia de un rol activo en nuestro estudio que hace que los pacientes se concentren sólo en dos roles.

También es significativo el elevado número de pacientes que deseaba un rol pasivo y lo alcanzó.
Tanto la inexistencia de un rol activo como la preferencia de uno pasivo pueden estar influenciados, en parte, por el modelo paternalista aún existente en España ${ }^{15,16,18,19}$ y están en la misma línea del estudio de la Fundació Josep Laporte y la Universitat Autònoma de Barcelona ${ }^{19}$, que confirma que los pacientes oncológicos no muestran gran interés en participar en las decisiones que afectan a su salud, y se apunta, igualmente, al paternalismo sanitario, aún mayoritario en la sanidad española, entre las posibles causas de dicha actitud.

Junto al paternalismo sanitario, pensamos que nuestros resultados también pueden verse influenciados por el hecho 
de que no todos los enfermos están preparados o desean tener el mismo grado de implicación o participación.

En lo referente a la medición del proceso de TDC, se encontró que los pacientes urológicos tenían una puntuación transformada según el SDM-Q-9 superior al resto de pacientes, concretamente de 61,39 $\pm 13,24$. En concreto consideraban que se cumplían en mayor medida todos los pasos de la TDC, salvo el 7 relativo a la negociación y el 9 referente al acuerdo sobre el modo de proceder. Sin embargo, el alcance del estudio no ha permitido determinar los motivos de la valoración superior concedida por los pacientes urológicos.

Por lo que respecta a la orientación de las respuestas del SDM-Q-9 ítem por ítem, hemos constatado que existe desacuerdo en 8 de las 9 afirmaciones del cuestionario en todos los pacientes. Pensamos, nuevamente, que éste puede estar motivado por el paternalismo (de los 73 pacientes que adoptaron un rol pasivo, 25 fueron por iniciativa propia y 48 no), así como por las barreras que influyen para la puesta en práctica de la TDC como la presión del tiempo, las características del paciente o la falta de incentivos del sistema actual para fomentar su utilización.

Entre las limitaciones de nuestro estudio podemos destacar, en primer lugar, que las conclusiones no se pueden generalizar debido a que la investigación se ha realizado en un único hospital, afectando a la representatividad de la muestra. Además, y dado que no era posible conocer el número total de pacientes que, inicialmente, se había enfrentado a una TDC, en el estudio se ha trabajado con una muestra representativa establecida a criterio del personal sanitario.

Por otro lado, al realizar la encuesta no hemos tenido en cuenta la etapa de la enfermedad, el hecho de estar o no en tratamiento o si lo ha recibido, así como el estado emocional del enfermo, lo cual puede influir en sus respuestas así como en una participación menos activa. Además, no hemos considerado que los roles preferido y percibido, así como la toma de decisiones en sí se evaluaron en el mismo momento del tiempo ni tampoco el intervalo existente entre la toma de decisiones y la realización de cada encuesta. Al ser un estudio retrospectivo, es posible que nuestros resultados puedan estar algo sesgados por la distorsión de la memoria y/o reinterpretación.

Por último, destacar que la adaptación al español de The Control Preference Scales no ha sido validada y la versión española del cuestionario SDM-Q-9 ha sido validada, pero no en una muestra de pacientes con cáncer españoles.

\section{BIBLIOGRAFÍA}

1. Coulter A, Entwistle V, Gilbert D. Sharing decisions with patients: is the information good enough? BMJ 1999; 318: 318-322.

2. President's Commission. President's Commission for the Study of Ethical Problems in Medicine and Biomedical and Behavioral Research. Making Health Care Decisions. The Ethical and Legal Implications of Informed Consent in the Patient-Practitioner Relationship. Washington DC: 1982.

3. Ernst J, Kuhnt S, Schwarzer A, Aldaoud A, NieDERWIESER D, MANTOVANI-LÖFFLER L et al. The desire for shared decision making among patients with solid and hematological cancer. Psychooncology 2011; 20: 186-193.

4. Hack TF, Degner LF, Watson P, Sinha L. Do patients benefit from participating in medical decision making? Longitudinal follow-up of women with breast cancer. Psychooncology 2006; 15: 9-19.

5. Kane HL, Halpern MT, Squiers LB, Treiman KA, McCormack LA. Implementing and evaluating shared decision making in oncology practice. CA Cancer J Clin 2014; 64: 377-388.

6. IzQuierdo F, Gracia J, Guerra M, Blasco JA, ANDRADAS E. Health technology assessment-based development of a Spanish breast cancer patient decision aid. Int $\mathrm{J}$ Technol Assess Health Care 2011; 27: 363-368.

7. Consejería de Salud de la Junta de Andalucía. Alternativas de tratamiento para el cáncer de próstata ¿Qué opción prefiero? Instrumento de Ayuda para la Toma de Decisiones. Dirección General de Calidad, Investigación y Gestión del Conocimiento, Granada, 2007.

8. Chewning B, Bylund CL, Shah B, Arora NK, GueGUEN JA, MAKoul G. Patient preferences for shared decisions: a systematic review. $\mathrm{Pa}$ tient Educ Couns 2012; 86: 9-18. 
9. Brom L, Hopmans W, Pasman HR, Timmermans DR, Widdershoven GA, OnwUteaka-PhILIPSEN BD. Congruence between patients' preferred and perceived participation in medical decisionmaking: a review of the literature. BMC Med Inform Decis Mak 2014; 14: 25.

10. Tariman JD, Berry DL, Cochrane B, Doorenbos A, SCHEPP K. Preferred and actual participation roles during health care decision making in persons with cancer: a systematic review. Ann Oncol 2010; 21: 1145-1151.

11. Vogel BA, Leonhart R, Helmes AW. Communication matters: the impact of communication and participation in decision making on breast cancer patients' depression and quality of life. Patient Educ Couns 2009; 77 : 391-397.

12. Atherton PJ, Smith T, Singh JA, Huntington J, Diekmann BB, HuschKa M, et al. The relation between cancer patient treatment decisionmaking roles and quality of life. Cancer 2013; 119: 2342-2349.

13. Scholl I, Koelewijn-van Loon M, Sepucha K, ElWyn G, LÉGaré F, Härter M et al. Measurement of shared decision making - a review of instruments. Z Evid Fortbild Qual Gesundhwes 2011; 105: 313-324.

14. Perestelo-Pérez L, Rivero-Santana A, Pérez-Ramos J, González-Lorenzo M, Román JG, SerranoAguilar P. Shared decision making in Spain: current state and future perspectives. Z Evid Fortbild Qual Gesundhwes 2011; 105: 289-295.

15. Barca Fernández I, Parejo Miguez R, Gutiérrez Martín P, Fernández Alarcón F, Alejandre Lázaro G, LÓPEZ DE CASTRo F. La información al paciente y su participación en la toma de decisiones clínicas. Aten Primaria 2004; 33: 361-364.

16. Peralta L. La participación del paciente en la toma de decisiones en las consultas de atención primaria. Tesis doctoral. Universidad de Cantabria. 2010.

17. Delgado A, López-Fernández LA, Luna J, Saletti Cuesta L, Gil Garrido N, Puga González A. Expectativas de los pacientes sobre la toma de decisiones ante diferentes problemas de salud. Gac Sanit 2010; 24: 66-71.

18. Ruiz Moral R, Peralta Munguia L, Pérula de TorRes LA, Olloqui Mundet J, Carrión de la Fuente T, Sobrino López A et al. Opiniones y percepciones de los pacientes sobre su participación en la toma de decisiones en las consultas de medicina de familia. Aten Primaria 2012; 44: 5-10.

19. Fundació Josep Laporte y Universitat Autònoma de Barcelona. El valor terapéutico en oncología. La perspectiva de los pacientes, familiares y profesionales. Publicaciones
Universidad de los Pacientes, Barcelona, 2009.

20. De las Cuevas C, Peñate W, Perestelo-Pérez L, Serrano-Agullar P. Shared decision making in psychiatric practice and the primary care setting is unique, as measured using a 9-item Shared Decision Making Questionnaire (SDM-Q-9). Neuropsychiatr Dis Treat 2013; 9: 1045-1052.

21. Charles CA, Whelan T, Gafni A, Willan A, FaRRELL S. Shared treatment decision making: what does it mean to physician? J Clin Oncol 2003; 21: 932-936.

22. López-Abente G, Aragonés N, Pérez-Gómez B, Pollán M, García-Pérez J, Ramis R, et al. Time trends in municipal distribution patterns of cancer mortality in Spain. BMC Cancer 2014; 14: 535.

23. Degner LF, Sloan JA, Venkatesh P. The control preferences scale. Can J Nurs Res 1997; 29: 21-43.

24. Wallberg B, Michelson H, Nystedt M, Bolund C, DEgner LF, WILKING N. Information needs and preferences for participation in treatment decisions among Swedish breast cancer patients. Acta Oncol 2000; 39: 467-476.

25. Kriston L, Scholl I, Hölzel L, Simon D, LoH A, HÄrTER M. The 9-item Shared Decision Making Questionnaire (SDM-Q-9). Development and psychometric properties in a primary care sample. Patient Educ Couns 2010; 80: 94-99.

26. De Las Cuevas C, Perestelo-Pérez L, RiveroSantana A, Cebolla-Martí A, Scholl I, Härter M. Validation of the Spanish version of the 9-item shared decision-making questionnaire. Health Expect 2015; 18: 2143-2153.

27. Bruera E, Sweeney C, Calder K, Palmer L, BeNISCH-Tolley S. Patient preferences versus physician perceptions of treatment decisions in cancer care. J Clin Oncol 2001; 19: 2883-2885.

28. Bruera E, Willey JS, Palmer JL, Rosales M. Treatment decisions for breast carcinoma: patient preferences and physician perceptions. Cancer 2002; 94: 2076-2080.

29. Singh JA, Sloan JA, Atherton PJ, Smith T, Hack TF, HuschKa MM et al. Preferred roles in treatment decision making among patients with cancer: a pooled analysis of studies using the Control Preferences Scale. Am J Manag Care 2010; 16: 688-696.

30. Colley A, Halpern J, Paul S, Micco G, Lahiff M, Wright F et al. Factors associated with oncology patients' involvement in shared decision making during chemotherapy. Psychooncology 2016 Sep 20. doi: 10.1002/ pon.4284. 
